\title{
Randomised controlled trial of intensive multifactorial treatment for cardiovascular risk in patients with screen-detected type 2 diabetes: 1-year data from the ADDITION Netherlands study
}

\author{
Paul GH Janssen, Kees J Gorter, Ronald P Stolk and Guy EHM Rutten
}

\author{
ABSTRACT \\ Background \\ A growing body of evidence suggests that earlier \\ diagnosis and treatment of diabetes may be beneficial; \\ however, definitive evidence is lacking. \\ Aim \\ To evaluate the effectiveness of an intensified \\ multifactorial treatment on cardiovascular risk factors in \\ patients with screen-detected type 2 diabetes.

\section{Design of study} \\ Randomised controlled trial.

\section{Setting} \\ Seventy-nine general practices in the southwestern \\ region of the Netherlands. \\ Method \\ In this randomised trial, patients diagnosed with \\ diabetes by screen-detection were assigned to \\ intensified ( $n=255)$ or routine treatment $(n=243)$, and \\ followed over 1 year. Intensified treatment consisted of \\ pharmacological treatment combined with lifestyle \\ education to achieve haemoglobin $\mathrm{A} 1 \mathrm{c}(\mathrm{HbA} 1 \mathrm{c})$ \\ $<7.0 \%$, blood pressure $<135 / 85 \mathrm{mmHg}$, and \\ cholesterol $<5.0 \mathrm{mmol} / \mathrm{l}(4.5 \mathrm{mmol} / \mathrm{l}$ if cardiovascular \\ disease was present). Health-related quality of life \\ (HRQoL) was assessed using the Short Form (SF)-36. \\ Analyses were performed using generalised estimating \\ equations models. \\ Results \\ Changes in body mass index were 0.2 (routine care) \\ versus $-1.4 \mathrm{~kg} / \mathrm{m}^{2}$ (intensified treatment), $P<0.001$; \\ systolic blood pressure -19 versus $-33 \mathrm{mmHg}$, \\ $P<0.001$; diastolic blood pressure -7 versus $-12 \mathrm{mmHg}$, \\ $P<0.001$; $\mathrm{HbA1c}-0.9 \%$ versus $-1.1 \%, P=0.03$; \\ cholesterol -0.5 versus $-1.2 \mathrm{mmol} / \mathrm{l}, P<0.001$; high- \\ density lipoprotein cholesterol 0.1 versus $0.1 \mathrm{mmol} / \mathrm{l}, P$ \\ $=0.26$; low-density lipoprotein cholesterol -0.5 versus \\ $-1.0 \mathrm{mmol} / \mathrm{l}, P<0.001$; triglycerides -0.3 versus \\ $-0.4 \mathrm{mmol} / \mathrm{l}, P=0.71$. No difference in $\mathrm{HRQ}$ oL between \\ the two groups was reported.

\section{Conclusion} \\ Intensified multifactorial treatment of patients with \\ screen-detected diabetes in general practice reduces \\ cardiovascular risk factor levels significantly without \\ worsening HRQoL. \\ Keywords \\ cardiovascular disease; primary health care; randomised \\ controlled trial; screening; diabetes mellitus, type 2.
}

\section{INTRODUCTION}

Patients with type 2 diabetes are at high risk of developing cardiovascular diseases (CVDs) and, consequently of reduced life expectancy. Type 2 diabetes may remain undiagnosed for many years, and at diagnosis complications are frequently present. ${ }^{1,2}$ There is increasing evidence that early detection and treatment of type 2 diabetes is beneficial, although definitive studies are lacking.,

To improve the prognosis of people with type 2 diabetes, prevention of CVD is needed. Intensive treatment of hyperglycaemia, hypertension, and dyslipidaemia can reduce the CVD risk. ${ }^{5-7}$ It has been demonstrated that multifactorial intervention in patients with diabetes with microalbuminuria slows cardiovascular and microvascular complications. ${ }^{8,9}$ However, it has not been investigated whether intensified, multifactorial treatment of patients with screen-detected diabetes can reduce CVD mortality and the incidence of macro- and microvascular complications. To this purpose, the ADDITION study (Anglo-Danish-Dutch Study of Intensive Treatment in People with Screen-Detected Diabetes in Primary Care) has been initiated. ${ }^{10}$ Patients with diabetes

PGH Janssen, MD, PhD, GP; KJ Gorter, MD, PhD, GP; GEHM Rutten, MD, PhD, professor of primary care, Julius Center for Health Sciences and Primary Care, University Medical Center, Utrecht; RP Stolk, MD, PhD, professor of clinical epidemiology, Department of Epidemiology, University Medical Center, Groningen, the Netherlands.

Address for correspondence

Paul Janssen, Julius Center for Health Sciences and Primary Care, University Medical Center Utrecht, Stratenum 6.101, PO Box 85060, 3508 AB Utrecht, the Netherlands. E-mail: pjanssen@nlzorg.net

Submitted: 12 May 2008; Editor's response: 1 July 2008; final acceptance: 16 September 2008.

OBritish Journal of General Practice 2009; 59: 43-48.

DOI: 10.3399/bjgp09X394851 


\section{How this fits in}

Early detection and treatment of type 2 diabetes might be beneficial, although

conclusive evidence is not available. Intensified multifactorial treatment of patients with screen-detected type 2 diabetes in general practice is feasible and reduces the levels of cardiovascular risk factors significantly. At end of this study no differences in health-related quality of life between the intensively treated group and the routine care group were found.

\section{GPs and practices}

Approximately 500 GPs in the region, all cooperating with one regional laboratory (SHL Centre for Diagnostic Support in Primary Care, Etten-Leur, the Netherlands), were invited to participate with an informative letter about the study. Subsequently, all GPs were contacted by telephone. Inclusion criteria for the participating GPs were attendance at an instruction meeting, agreement that all GPs in the practice or partnership would participate in the ADDITION study, and agreement to treat all patients with screen-detected diabetes according to the randomly allocated treatment protocol. A total of 79 practices were randomly selected from those willing to participate. Randomisation was performed according to stratification of practice organisation (single-handed, group practices).

In $41 \%$ of the practices in the intensified treatment group, and in $52 \%$ of the practices in the routine care group, practice nurses were involved in daily diabetes care. care according to the guidelines of the Dutch College of General Practitioners. ${ }^{12}$

\section{METHOD \\ ADDITION study}

This study is part of the international ADDITION study which is a randomised controlled intervention trial of 3057 patients with screen-detected diabetes. ${ }^{10,13}$ ADDITION consists of a screening study and a subsequent intervention trial with a follow-up of 5 years. In the intervention trial the effects of routine care in general practice according to national guidelines are compared with those of an intensified, multifactorial treatment on mortality and cardiovascular morbidity.

In the ADDITION Netherlands study, from 2002 to 2004, a total of 56978 non-diabetic registered patients aged $50-70$ years from 79 general practices in the southwestern region of the Netherlands were invited to participate in a stepwise population-based screening programme. The screening algorithm has been described in detail elsewhere. ${ }^{14}$ The screening started with a self-completed questionnaire based on a validated risk score..$^{15}$ Participants with a risk score above threshold were invited for further diagnostic testing. Diagnosis of diabetes was based on the 1999 World Health Organization definition. ${ }^{16}$ In total, 586 new patients with diabetes were detected. ${ }^{14}$

\section{Study design}

ADDITION Netherlands is a multipractice trial with practice-level randomisation to intensified treatment or routine care. Patients were blinded to which treatment arm their GP had been randomised to. The intensive treatment protocol was carried out by a diabetes nurse together with a GP.

\section{Patients}

In the Netherlands, the entire population is registered with a GP. Therefore, the screened population may be considered to be a representative sample of the full population. The invitation letter was sent to the patients' home addresses and signed by their own GP. The letter explained the aim of the study; also enclosed with the letter were an informed consent form and a questionnaire for self-completion which contained questions about age, sex, body mass index, family history of diabetes, frequent thirst, use of antihypertensive medication, shortness of breath, claudication, and exercise such as cycling.

Exclusion criteria were any contraindications to or history of major intolerance to any of the drugs used in the study; a history of alcoholism, drug abuse, psychosis, personality disorder, or another emotional, psychological, or intellectual problem that is likely to comply with the protocol requirements; and/or being treated for a malignant disease or otherwise having a poor prognosis.

\section{Details of the intervention}

Intensively treated group. During a 3-hour session, GPs were trained in the treatment protocol. This protocol is characterised by intensive treatment of glucose, blood pressure, and lipids, and structured lifestyle education (dietary modification, weight loss, increased physical activity, smoking cessation and improving adherence to medication).

Haemoglobin A1c (HbA1c) level should be kept under $7.0 \%$. Alternations or additions to glucoselowering therapy should already be initiated when invalidate informed consent or limit the ability to 
$\mathrm{HbA} 1 \mathrm{c}>6.5 \%$. If $\mathrm{HbA} 1 \mathrm{c}$ remains above $7.0 \%$ with oral agents, insulin therapy should be initiated. Antihypertensive agents were prescribed if blood pressure was $>120 / 80 \mathrm{mmHg}$ (systolic or diastolic). Angiotensin-converting enzyme (ACE) inhibitors were the initial treatment unless there were side-effects. In that case an angiotensin-II receptor antagonist was prescribed. If blood pressure was $>135 / 85 \mathrm{mmHg}$, the dose had to be increased, and calcium channel blockers, thiazides, or beta-blockers were added with a stepwise approach. Treatment with a statin was indicated if cholesterol was $>5.0 \mathrm{mmol} / / \mathrm{l}$ or $>4.5 \mathrm{mmol} / \mathrm{l}$ in patients without or with a known history of CVD respectively. The dose of statin was increased up to maximum if cholesterol remained above threshold. In 2003 the protocol changed: all participants with cholesterol $>3.5 \mathrm{mmol} / \mathrm{l}$ were treated with lipidlowering drugs. Acetylsalicylic acid $80 \mathrm{mg}$ per day was given to patients treated with antihypertensive agents.

Diabetes nurses were trained in the management of the treatment algorithms and in providing lifestyle education. They were authorised to prescribe medication, supervised by the GPs. During the first year of the intervention, every 3 months a 2-hour training session was arranged. Objectives of these sessions included discussing obstacles in reaching the target values, exchanging experiences, and evaluating cooperation with GPs. At least once per year, GPs from the intervention group were reminded about treating their ADDITION patients according to the protocol.

Patients diagnosed with type 2 diabetes were first seen by their GP. Subsequently, visits were paid to the diabetes nurse at least 2, 4, 6, 8, and 12 weeks after diagnosis until target levels were reached. Thereafter, patients were requested to visit the GP or nurse every 3 months. One year after screening, an extensive visit to the GP took place. Patients were referred to an internist if the diabetes nurse and GP did not succeed in reaching the targets.

Routine care group. According to the 1999 guidelines from the Dutch College of General Practitioners, ${ }^{12}$ the target value for $\mathrm{HbA} 1 \mathrm{c}$ was $<7.0 \%$. However, in the guidelines $\mathrm{HbA} 1 \mathrm{c}$ levels between $7.0 \%$ and $8.5 \%$ were described as acceptable. Lipid-lowering drugs should be initiated if participants without a known history of CVD had a risk above $25 \%$ of developing a coronary disease within 10 years. Participants with previous CVD should be treated with lipid-lowering medication if cholesterol $>5.0 \mathrm{mmol} / \mathrm{l}$. If blood pressure was $>150 / 85 \mathrm{mmHg}$, treatment was recommended starting with a thiazide diuretic. Subsequently, an ACE inhibitor or a beta-blocker could be added, or the combination of thiazide, ACE inhibitors, and beta-blockers was given. Detailed instructions about providing lifestyle education were not given. ${ }^{12}$ After the initiation symposium, no further training of GPs was provided.

\section{Measurements}

Blood pressure was measured using an Omron M4 blood pressure recorder (Omron Healthcare, Milton Keynes, UK). Fasting blood glucose was determined with a HemoCue B-Glucose Analyser based on the glucose-dehydrogenase method (HemoCue,

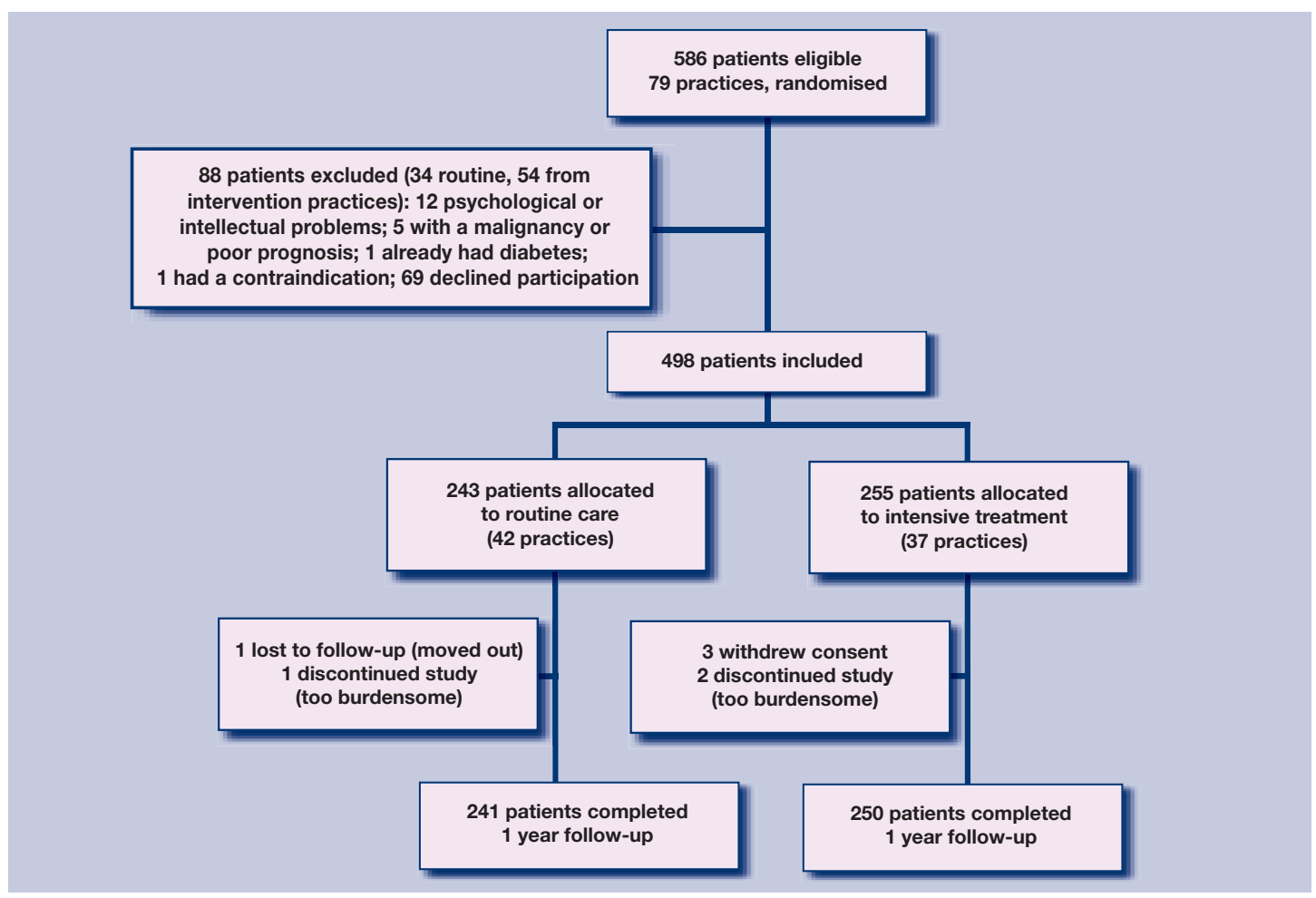

Figure 1. Trial profile. 
Angelholm, Sweden). $\mathrm{HbA1c}$ was measured with highperformance liquid chromatography (160 A. Menarini Diagnostics, Firenze, Italy). Lipids were determined with enzymatic techniques (LX20 Beckman, Fullerton, CA, US). All biochemical tests were performed in the regional laboratory.

Laboratory results were extracted from the regional laboratory where all patients were examined regularly, independent of intervention arm. Blinded data collection was done by research assistants. In the routine care group, data were derived from patients' files at the practices, and in the intervention group data were derived from case record forms.

Prior to entry into the study, participants completed the Short Form (SF)-36, a validated instrument designed for the self-evaluation of health-related quality of life (HRQoL). ${ }^{17,18}$ The SF-36 measures general health, vitality, mental health, physical functioning, limitations due to physical difficulties (role physical),

\section{Table 1. Baseline characteristics and changes in biomedical variables after 1 year in both treatment groups.}

\begin{tabular}{|c|c|c|c|c|c|}
\hline & \multicolumn{2}{|c|}{$\begin{array}{l}\text { Routine care } \\
(n=243)\end{array}$} & \multicolumn{2}{|c|}{$\begin{array}{l}\text { Intensive treatment } \\
(n=255)\end{array}$} & \multirow[t]{2}{*}{$P$-value ${ }^{a}$} \\
\hline & Baseline & 1 year & Baseline & 1 year & \\
\hline \multicolumn{6}{|l|}{ Demographic variables } \\
\hline Sex, \% male & 56.0 & & 51.8 & & \\
\hline Age in years, mean (SD) & $59.9(5.1)$ & & $60.1(5.4)$ & & \\
\hline Ethnicity, \% white & 98.7 & & 98.0 & & \\
\hline \multicolumn{6}{|l|}{ Behavioural variables } \\
\hline Current smoking, \% & 21.4 & & 26.3 & & \\
\hline Ever smoked, \% & 68.0 & & 66.1 & & \\
\hline Drinking alcohol, \% & 78.1 & & 78.4 & & \\
\hline Weekly $>5$ pieces of fruit, $\%$ & 61.7 & & 57.6 & & \\
\hline $\begin{array}{l}\text { Exercise (days per week } \\
>30 \mathrm{~min} \text { ), mean (SD) }\end{array}$ & $3.8(2.5)$ & & $4.0(2.3)$ & & \\
\hline \multicolumn{6}{|l|}{ Clinical variables, mean (SD) } \\
\hline Body mass index, $\mathrm{kg} / \mathrm{m}^{2}$ & $30.4(4.6)$ & $30.6(4.8)$ & $31.2(5.1)$ & $29.8(4.8)$ & $<0.001$ \\
\hline $\begin{array}{l}\text { Systolic blood pressure, } \\
\mathrm{mmHg}\end{array}$ & $163(23)$ & $144(17)$ & $166(23)$ & $133(17)$ & $<0.001$ \\
\hline $\begin{array}{l}\text { Diastolic blood pressure, } \\
\mathrm{mmHg}\end{array}$ & $89(10)$ & $82(8)$ & $90(11)$ & $78(9)$ & $<0.001$ \\
\hline \multicolumn{6}{|c|}{ Biochemical variables, mean (SD) } \\
\hline $\begin{array}{l}\text { Fasting blood glucose, } \\
\mathrm{mmol} / \mathrm{l}\end{array}$ & $8.1(2.8)$ & $7.2(1.7)$ & $7.8(2.3)$ & $6.5(1.1)$ & 0.02 \\
\hline $\mathrm{HbA} 1 \mathrm{c}, \%$ & $7.4(1.7)$ & $6.5(0.9)$ & $7.3(1.6)$ & $6.2(0.6)$ & 0.03 \\
\hline Cholesterol, mmol/l & $5.6(1.1)$ & $5.1(1.0)$ & $5.6(1.1)$ & $4.4(0.9)$ & $<0.001$ \\
\hline HDL-cholesterol, mmol/l & $1.1(0.3)$ & $1.2(0.9)$ & $1.1(0.4)$ & $1.1(0.3)$ & 0.26 \\
\hline LDL-cholesterol, mmol/l & $3.7(1.0)$ & $3.2(1.0)$ & $3.7(1.0)$ & $2.7(0.8)$ & $<0.001$ \\
\hline Triglycerides, mmol/l & $2.0(1.6)$ & $1.7(1.7)$ & $1.9(1.0)$ & $1.5(0.8)$ & 0.71 \\
\hline
\end{tabular}

Practices, \%

Single-handed $\quad 50.0 \quad 43.2$

$\begin{array}{lll}\text { Urban location } & 52.4 & 29.7\end{array}$

Specific diabetes clinic $\quad 63.4 \quad 62.2$

$>10 \%$ patients from ethnic $\quad 7.1 \quad 8.1$

minority groups

aComparison of changes in variables between treatment groups adjusted for age, sex, baseline value, and clustering at practice level. $\mathrm{HbA1c}=$ Haemoglobin $\mathrm{A1c} . \mathrm{HDL}=$ high-density lipoprotein. $L D L=$ low-density lipoprotein. bodily pain, social functioning, and limitations due to emotional difficulties (role emotional). For each dimension, scores range from 0 (worst health) to 100 (best health).

\section{Statistical analyses}

Analyses were performed using generalised estimating equations models, adjusting for age, sex, baseline values, and clustering at practice level.

SF-36 scores at the end of the study were analysed using analysis of covariance with age, sex, and baseline values as covariates to adjust for differences between treatment groups at randomisation. Pairedsamples $t$-tests were used to compare baseline with follow-up within each treatment group.

Analyses were based on intention-to-treat. Last available measurements were used for participants who were lost to follow-up, and for missing data. $P$ value $<0.05$ was considered statistically significant. Data were analysed using the SPSS statistical package (version 15.0). The CONSORT checklist for cluster randomised controlled trials was adhered to.

\section{RESULTS}

Of the 586 patients with screen-detected diabetes, 498 were included. A total of 255 participants were assigned to intensified treatment and 243 to routine care. Excluded from the study were 88 people: 69 declined participation, 12 had psychological or intellectual problems, five had a poor prognosis, one person already had diabetes, and one had a contraindication.

The trial profile is shown in Figure 1. Of all 498 included patients, seven (two in the routine care group, five in the intensively treated group) were lost to followup for various reasons (relocation, withdrawal of consent, treatment too burdensome). Reasons for study discontinuation were similar between both treatment groups.

The two groups were well matched with respect to clinical, biochemical, and behavioural characteristics (Table 1), as well as use of cardiovascular medications and history of cardiovascular events (data not shown). With the exception of high-density lipoprotein (HDL)cholesterol and triglycerides, variables improved significantly better after 1 year in the intensively treated group compared with the routine care group. One hypoglycaemic event (requiring assistance) occurred in the intervention group.

SF-36 scores between both treatment groups at the end of the study were not significantly different (Table 2). Within both treatment groups, general health, vitality and mental health, and in the intensively treated group also physical functioning, improved significantly during follow-up. Scores on social functioning decreased equally in both groups. 


\begin{tabular}{|c|c|c|c|c|c|}
\hline & \multicolumn{2}{|c|}{$\begin{array}{l}\text { Routine care } \\
\qquad(n=243)\end{array}$} & \multicolumn{2}{|c|}{$\begin{array}{l}\text { Intensive treatment } \\
\quad(n=255)\end{array}$} & \multirow[t]{2}{*}{$\begin{array}{c}\text { Difference between groups } \\
P \text {-value }\end{array}$} \\
\hline & Baseline & 1 year & Baseline & 1 year & \\
\hline General health & $59.7(12.0)$ & $64.4(18.1)^{b}$ & $59.1(11.5)$ & $63.3(18.4)^{b}$ & 0.632 \\
\hline Vitality & $52.2(13.2)$ & $67.1(18.4)^{\mathrm{b}}$ & $49.3(14.4)$ & $64.8(20.4)^{\mathrm{b}}$ & 0.814 \\
\hline Mental health & $69.9(12.6)$ & $79.0(15.6)^{b}$ & $68.4(13.3)$ & $75.9(17.9)^{b}$ & 0.559 \\
\hline Physical functioning & $78.3(22.0)$ & $78.1(23.2)$ & $77.4(21.9)$ & $80.1(21.2)^{b}$ & 0.218 \\
\hline Role physical & $84.9(30.0)$ & $81.1(33.5)$ & $82.8(31.4)$ & $80.3(35.0)$ & 0.930 \\
\hline Bodily pain & $84.7(20.7)$ & $82.2(22.4)$ & $80.8(22.1)$ & $79.2(22.7)$ & 0.970 \\
\hline Social functioning & $89.0(17.2)$ & $85.7(19.2)^{\mathrm{b}}$ & $87.9(20.0)$ & $83.0(22.0)^{\mathrm{b}}$ & 0.368 \\
\hline Role emotional & $85.4(32.4)$ & $89.9(26.0)$ & $88.2(28.6)$ & $86.2(30.9)$ & 0.254 \\
\hline
\end{tabular}

\section{DISCUSSION}

\section{Summary of main findings}

In this randomised controlled trial, patients with screen-detected type 2 diabetes in general practice were followed for 1 year. In patients assigned to intensified multifactorial treatment, clinical and biochemical variables improved significantly more than in those who received routine care. The study findings showed cardiovascular risk factors to be considerably modifiable by an intensified, multifactorial treatment in a population at high risk for developing cardiovascular complications. At the end of follow-up, SF-36 scores were similar for the two arms, suggesting no major detrimental impact on quality of life from the intensive intervention.

\section{Strengths and limitations of the study}

Some limitations should be taken into account in interpreting the results of this study. Patients in the intensive arm were treated by diabetes nurses who had enough time to motivate patients. Therefore, it might be disputed whether the treatment targets achieved in this trial are also achievable in daily practice. However, in many general practices, specialised nurses are already involved in daily diabetes care, making the implementation of an intensified treatment feasible. GPs in the routine care practices followed the 1999 Dutch guidelines on type 2 diabetes. ${ }^{12}$ These guidelines were updated in 2006. It should be emphasised that it is not possible to rule out that in advance of the 2006 guidelines these GPs might already have tended towards tighter control for cardiovascular risk factors. As a result, the impressive differences in biomedical variables between both treatment groups after 1 year of treatment might become less so later on. Numbers of missing 1-year data were comparable between both groups. Analyses were based on intention-to-treat, with the last value carried forward for missing data. Given the strong decrease in cardiovascular risk factor levels in the intervention arm, the decrease might be even greater when no data would have been missing. In the routine care group, considerably more urban practices were included. However, great similarities were found between baseline cardiovascular risk factor levels in urban and rural practices (data not shown).

A strength of this trial is that the study population was unselected. To the authors' knowledge, this is the first randomised trial on the effects of an intensive, multifactorial intervention in patients with type 2 diabetes identified by screening. Participants in landmark trials on cardiovascular risk, such as the Heart Protection Study, the Antihypertensive and LipidLowering Treatment to Prevent Heart Attack Trial, the UK Prospective Diabetes Study, the ADVANCE study, or the ACCORD trial, were not intensively treated for all known cardiovascular risk factors together. ${ }^{19-23}$

\section{Comparison with existing literature}

Another randomised trial in general practice to assess the effect of a multifaceted intervention was carried out in Denmark. ${ }^{24}$ This study included clinically diagnosed patients with type 2 diabetes. GPs were assigned to routine care or structured care. In the intervention group, GPs were supported by prompting, feedback, and clinical guidelines to reach individualised targets. After 6 years of follow-up, differences between both groups were seen for fasting plasma glucose, HbA1c, and systolic blood pressure, but not for smoking habits, exercise, diastolic blood pressure, cholesterol, and triglycerides. At the end of the study, no differences were found in occurrence of myocardial infarction, stroke, or diabetic retinopathy. The treatment goals in this trial were less ambitious than those in the ADDITION study. In the Steno type 2 study, in which diabetic patients with microalbuminuria were included, cardiovascular event rate was cut by half in the intensively treated group (mean follow-up 7.8 years). ${ }^{8,9}$ Nevertheless, the event rate was 
considerable (3.5\%) in this high-risk population.

Improvements in SF-scores found in the present study were not seen in the Dutch Hoorn study which included patients with screen-detected diabetes, aged 50-75 years, who received routine care after screening. Baseline and 1-year SF-36 scores in the Hoorn study did not differ substantially, except for the dimension 'role emotional'. ${ }^{25}$ In the US, HRQoL scores at baseline and after 1 year were similar for persons with and without diabetes found at screening, and remained practically unaltered over the year after screening. ${ }^{26}$

SF-36 scores between both treatment groups at the end of the study were not different. With two groups of 250 patients, it is possible to detect a difference of 5.0 between the SF-36 scores (standard deviation 20, power $80 \%$, two-sided alpha 0.05 ). This means that subtle differences in SF-36 scores cannot be detected with the present study population.

\section{Implications for future research and clinical practice}

The results of this study indicate that an intensified, multifactorial treatment for patients with screendetected type 2 diabetes in general practice is feasible, at least in the short term, and reduces the levels of cardiovascular risk factors significantly without worsening HRQoL. Final results of the ADDITION study in 2010 must be awaited to evaluate the beneficial effect of this intensified intervention on the development of cardiovascular events in patients with screen-detected diabetes. The primary endpoint for the 5-year follow-up will be a composite cardiovascular outcome comprising cardiovascular mortality and morbidity (myocardial infarction, non-fatal stroke), revascularisations, and amputations. ${ }^{13}$

\section{Funding body}

The ADDITION Netherlands study is made possible by unrestricted grants from NovoNordisk, Glaxo Smith Kline, and Merck. Trial registry number is ClinicalTrials.gov ID no: NCT 00237549

\section{Ethical approval}

The study was approved by the Medical Ethical Committee of the University Medical Centre Utrecht, the Netherlands

\section{Competing interests}

The authors have stated that there are none

Acknowledgements

We wish to thank the directors, coordinating staff, and all diabetes nurses of the SHL Centre for Diagnostic Support in Primary Care, Etten-Leur, the Netherlands.

\section{Discuss this article}

Contribute and read comments about this article on the Discussion Forum: http://www.rcgp.org.uk/bjgp-discuss

\section{REFERENCES}

1. Harris MI, Klein R, Welborn TA, Knuiman MW. Onset of NIDDM occurs at least 4-7 years before clinical diagnosis. Diabetes Care 1992; 15(7): 815-819.

2. Rajala U, Laakso M, Qiao Q, Keinanen-Kiukaanniemi S. Prevalence of retinopathy in people with diabetes, impaired glucose tolerance and normal glucose tolerance. Diabetes Care 1998; 21(10): 1664-1669.

3. Borch-Johnsen K, Lauritzen T, Glümer C, Sandbaek A. Screening for type 2 diabetes — should it be now? Diabet Med 2003; 20(3): 175-181.
4. Colagiuri S, Cull CA, Holman RA. Are lower fasting plasma glucose levels at diagnosis of type 2 diabetes associated with improved outcomes? UKPDS 61. Diabetes Care 2002; 25: 1410-1417.

5. Coutinho M, Gerstein HC, Wang Y, Yusuf S. The relationship between glucose and incident cardiovascular events. A metaregression analysis of published data from 20 studies of 95783 individuals followed for 12.4 years. Diabetes Care 1999; 22(2): 233-240.

6. UK Prospective Diabetes Study Group. Tight blood pressure control and risk of macrovascular and microvascular complications in type 2 diabetes: UKPDS 38. BMJ 1998; 317(7160): 703-713.

7. Heart Protection Study Collaborative Group. MRC/BHF Heart Protection Study of cholesterol lowering with simvastatin in 5963 people with diabetes. Lancet 2003; 361(9374): 2005-2016.

8. Gaede P, Vedel P, Parving HH, Pedersen O. Intensified multifactorial intervention in patients with type 2 diabetes mellitus and microalbuminuria: the Steno type 2 randomised study. Lancet 1999; 353(9153): 617-622.

9. Gaede P, Vedel P, Larsen N, et al. Multifactorial intervention and cardiovascular disease in patients with type 2 diabetes. N Engl J Med 2003; 348(5): 383-393.

10. Lauritzen T, Griffin S, Borch-Johnsen K, et al. The ADDITION study: proposed trial of the cost-effectiveness of an intensive multifactorial intervention on morbidity and mortality among people with Type 2 diabetes detected by screening. Int J Obes Relat Metab Disord 2000; 24 (Suppl 3): S6-S11.

11. Adriaanse MC, Snoek FJ, Dekker JM, et al. Screening for type 2 diabetes: an exploration of subject's perceptions regarding diagnosis and procedure. Diabet Med 2002; 19(5): 405-411.

12. Rutten GEHM, Verhoeven S, Heine RJ, et al. Dutch College of General Practitioners. Practice guideline diabetes mellitus type 2 [in Dutch]. Huis Wetensch 1999; 42: 67-84.

13. Sandbaek A, Griffin SJ, Rutten G, et al. Stepwise screening for diabetes identifies people with high but modifiable coronary heart disease risk. The ADDITION study. Diabetologia 2008; 51(7): 1127-1134.

14. Janssen PGH, Gorter KJ, Stolk RP, Rutten GEHM. Low yield of population-based screening for Type 2 diabetes in the Netherlands: the ADDITION Netherlands study. Fam Pract 2007; 24(6): 555-661.

15. Ruige JB, de Neeling JND, Kostense PJ, et al. Performance of an NIDDM screening questionnaire based on symptoms and risk factors. Diabetes Care 1997; 20(4): 491-496

16. Alberti KGMM, Zimmet PZ. Definition, diagnosis and classification of diabetes mellitus and its complications. part 1: diagnosis and classification of diabetes mellitus. Geneva: World Health Organization, 1999.

17. Ware JE, Sherbourne CD. The MOS 36-item short-form health survey (SF36). I. Conceptual framework and item selection. Med Care 1992; 30(6): $473-483$.

18. McHorney CA, Ware JE Jr Lu JF Sherbourne CD. The MOS 36-item Short-Form Health Survey (SF-36): III. Tests of data quality, scaling assumptions, and reliability across diverse patient groups. Med Care 1994; 32(1): 40-66.

19. Heart Protection Study Collaborative Group. MRC/BHF Heart Protection Study of cholesterol lowering with simvastatin in 20,536 high-risk individuals: a randomised placebo-controlled trial. Lancet 2002; 360(9326): $7-22$.

20. ALLHAT Collaborative Research Group. Major outcomes in high-risk hypertensive patients randomized to angiotensin-converting enzyme inhibitor or calcium channel blocker vs diuretic. The Antihypertensive and Lipid-Lowering Treatment to Prevent Heart Attack Trial (ALLHAT). JAMA 2002; 288(23): 2981-2997.

21. UKPDS Group. Intensive blood glucose control with sulphonylureas or insulin compared with conventional treatment and risk of complications in patients with type 2 diabetes (UKPDS 33). Lancet 1998; 352(9131): 837-853.

22. ADVANCE Collaborative Group. Intensive blood glucose control and vascular outcomes in patients with type 2 diabetes. N Engl J Med 2008; 358(24): 2560-2972.

23. Action to Control Cardiovascular Risk in Diabetes Study Group. Effects of intensive glucose lowering in type 2 diabetes. N Engl J Med 2008; 358(24): 2545-2559.

24. Olivarius $\mathrm{N}$ de F, Beck-Nielsen $\mathrm{H}$, Andreasen $\mathrm{AH}$, et al. Randomised controlled trial of structured personal care of type 2 diabetes mellitus. BMJ 2001; 323(7319): 970-975.

25. Adriaanse MC, Snoek FJ, Dekker JM, et al. No substantial psychological impact of the diagnosis of Type 2 diabetes following targeted population screening: The Hoorn Screening Study. Diabet Med 2004; 21(9): 992-998.

26. Edelman D, Olsen MK, Dudley TK, et al. Impact of diabetes screening on quality of life. Diabetes Care 2002; 25(6): 1022-1026. 